\title{
Conséquences de l'instabilité morphodynamique liée à l'exploitation du soufre dans le massif volcanique du Calimani (Roumanie)
}

Consequences of the morphodynamic instability induced by the sulphur exploitation in the Călimani volcanic massif (Romania)

Die Folgen der morphodynamischen Instabilität im Bereich des Schwefelabbaus im Călimani-Gebirge (Rumänien)

Olimpiu Traian Pop, Nicolaie Hodor, Virgil Surdeanu et loan-Aurel Irimus

\section{OpenEdition \\ Journals}

Édition électronique

URL : http://journals.openedition.org/rge/955

DOI : $10.4000 /$ rge.955

ISSN : 2108-6478

Éditeur

Association des géographes de l'Est

Édition imprimée

Date de publication : 1 janvier 2009

ISSN : 0035-3213

\section{Référence électronique}

Olimpiu Traian Pop, Nicolaie Hodor, Virgil Surdeanu et loan-Aurel Irimus, « Conséquences de l'instabilité morphodynamique liée à l'exploitation du soufre dans le massif volcanique du Calimani (Roumanie) », Revue Géographique de l'Est [En ligne], vol. 49 / 1 | 2009, mis en ligne le 17 décembre 2009, consulté le 08 septembre 2020. URL : http://journals.openedition.org/rge/955 ; DOI : https:// doi.org/10.4000/rge.955

Ce document a été généré automatiquement le 8 septembre 2020

Tous droits réservés 


\title{
Conséquences de l'instabilité morphodynamique liée à l'exploitation du soufre dans le massif volcanique du Calimani (Roumanie)
}

\author{
Consequences of the morphodynamic instability induced by the sulphur \\ exploitation in the Călimani volcanic massif(Romania) \\ Die Folgen der morphodynamischen Instabilität im Bereich des Schwefelabbaus \\ im Călimani-Gebirge (Rumänien)
}

Olimpiu Traian Pop, Nicolaie Hodor, Virgil Surdeanu et Ioan-Aurel Irimus

Les auteurs tiennent à remercier vivement $M$. Wilfried Schreiber et Cristina POP d'avoir traduit le résumé en allemand et anglais, ainsi que le relecteur anonyme pour ses remarques constructives.

\section{Introduction}

1 Située dans la partie ouest des Carpates Orientales roumains, la chaîne volcanique néogène-quaternaire est constituée de plusieurs édifices volcaniques complexes dont le massif du Calimani (figure 1). Ce dernier représente est un massif volcanique composite, le plus étendu de toute la chaîne, culminant actuellement à plus de $2100 \mathrm{~m}$ d'altitude (le sommet du Pietrosul).

2 Sa genèse est liée à un contexte tectonique de subduction de plaques et à la formation d'un magma de composition principalement calco-alcaline. Une caldera volcanique d'environ $10 \mathrm{~km}$ de diamètre, des corps hypo-volcaniques intrusifs mis en relief par l'érosion à l'intérieur de cette caldera et plusieurs strato-cônes sont entourés par des plateaux volcanoclastiques externes caractérisant la morphologie du massif. Leur mise 
en place a été conditionnée par l'activité volcanique (explosive, extrusive, effusive, intrusive) et volcano-tectonique déroulées entre 11,9 et 6,7 MA (Pécskay et. al., 1995; Seghedi et al., 2005).

Figure 1 : Localisation du massif du Calimani (le rectangle blanc) dans le cadre de la Chaîne Volcanique des Carpates Roumaines

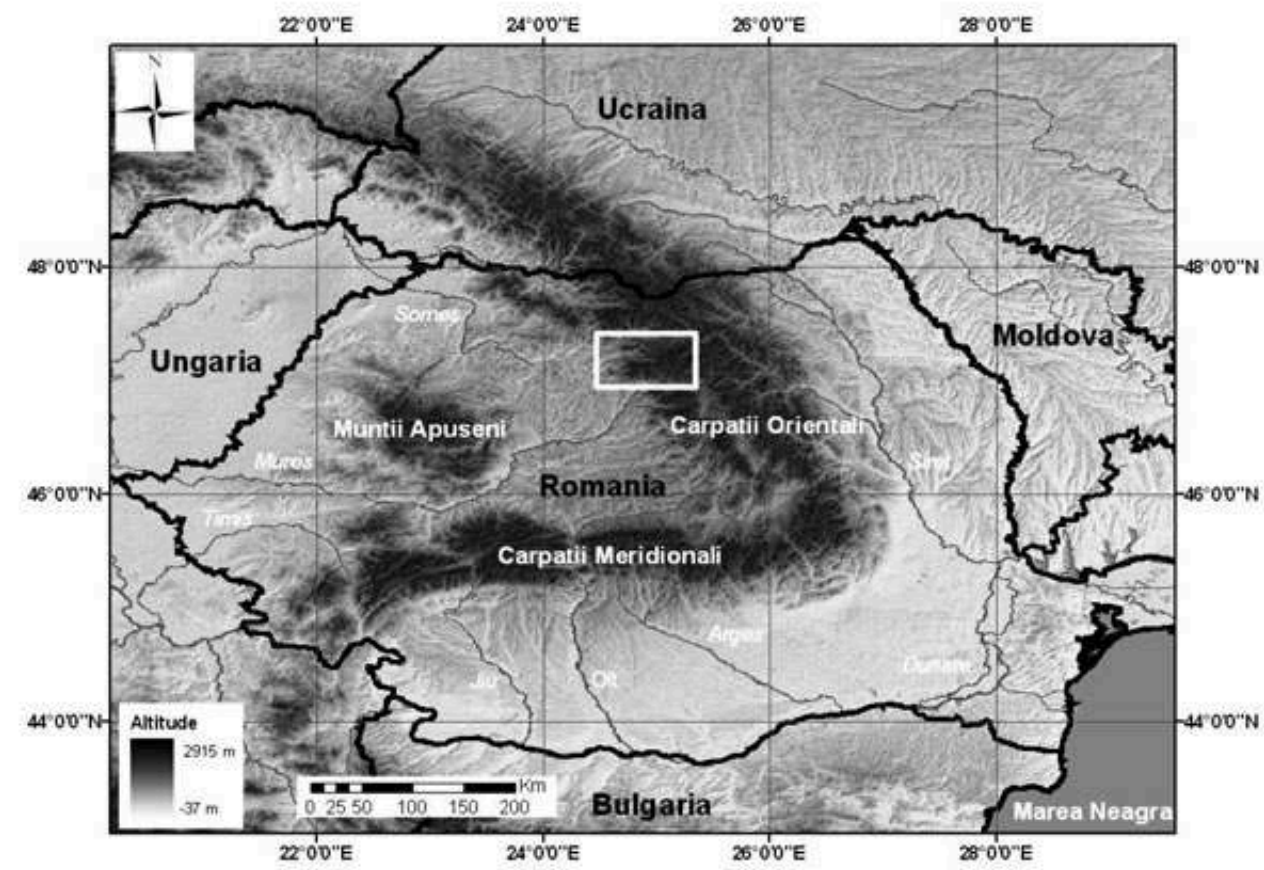

Les déstabilisations successives des flancs (Szakács et al., 2000, Szakács et Krézsek, 2006) et l'érosion intense des édifices volcaniques dans des contextes morphoclimatiques variés (subtropical, glacial et périglaciaire, actuel) ont modifié la morphologie volcanique initiale, le taux d'érosion du relief de 31,5 m / Ma, (Karátson, 1996) étant assez important.

4 L'activité post-volcanique intense caractérisée par des émanations de gaz (dioxyde de soufre-SO2, hydrogène sulfuré-H2S, etc.) à travers les fissures, leur interaction avec l'air atmosphérique et les eaux d'infiltration, ont conduit au dépôt du soufre par sublimation. Sur les flancs des cônes andésitique de Negoiul Românesc, 6,7 Ma et celui dacitique du Pietricelul, 7,2 Ma. (Pécskay et al., 1995), les prospections géologiques ont mis en évidence des dépôts pyroclastiques altérés contenant du soufre natif imprégné. Les laves andésitiques qui couvrent ces dépôts pyroclastiques et forment les sommets des cônes, présentent des fissures dans lesquelles le soufre s'est déposé. L'activité minière de prospection et d'exploitation des gisements contenant du soufre a transformé profondément la topographie de ces deux cônes (figure 2).

En ce qui concerne les conditions climatiques actuelles dans la carrière et les terrils, nous avons analysé les données météo, enregistrées à partir de 1990 à la station Retitiş. Celle-ci est située sur la bordure sud de la caldera, à l'altitude de $2021 \mathrm{~m}$ et à une distance d'environ $2 \mathrm{~km}$ de la carrière et des terrils (figure 2). Les valeurs des températures moyennes annuelles pour l'intervalle 1990-2007 sont présentées dans la figure 3. 
Figure 2 : Localisation des zones affectées par les activités minières dans le massif du Calimani (le rectangle blanc) : A - sommets; $B$ - cours d'eau permanent; $C$ - carrière, terrils, bâtiments.

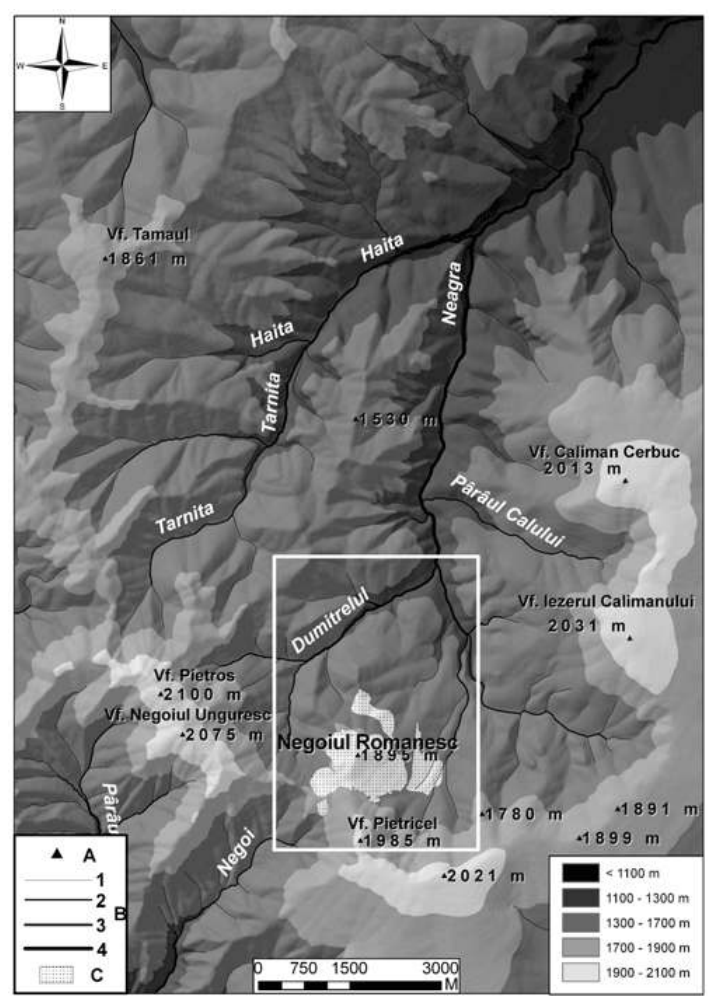

6 Sur le graphique de la figure 3, on observe qu'à l'altitude de l'étage alpin et subalpin les valeurs des températures moyennes annuelles sont autour de $0^{\circ} \mathrm{C}$. Dans ces conditions, la présence du couvert neigeux au sol et des sols gelés est prolongée pendant plus de la moitié de l'année, ce phénomène ayant des influences majeures sur la manifestation des processus géomorphologiques et sur leur fréquence. La période végétative des plantes en général et des arbres en particulier est raccourcie en fonction de la présence ou l'absence des taches de neige.

7 Le graphique de la figure 4 présente les sommes annuelles des précipitations (solides et liquides) calculées à partir des enregistrements journaliers effectués à la station Retitiş. 
Figure 3 : Les températures moyennes annuelles pour la période 1990-2007 sur les crêtes de la bordure sud de la caldera (station météo Retitiş).

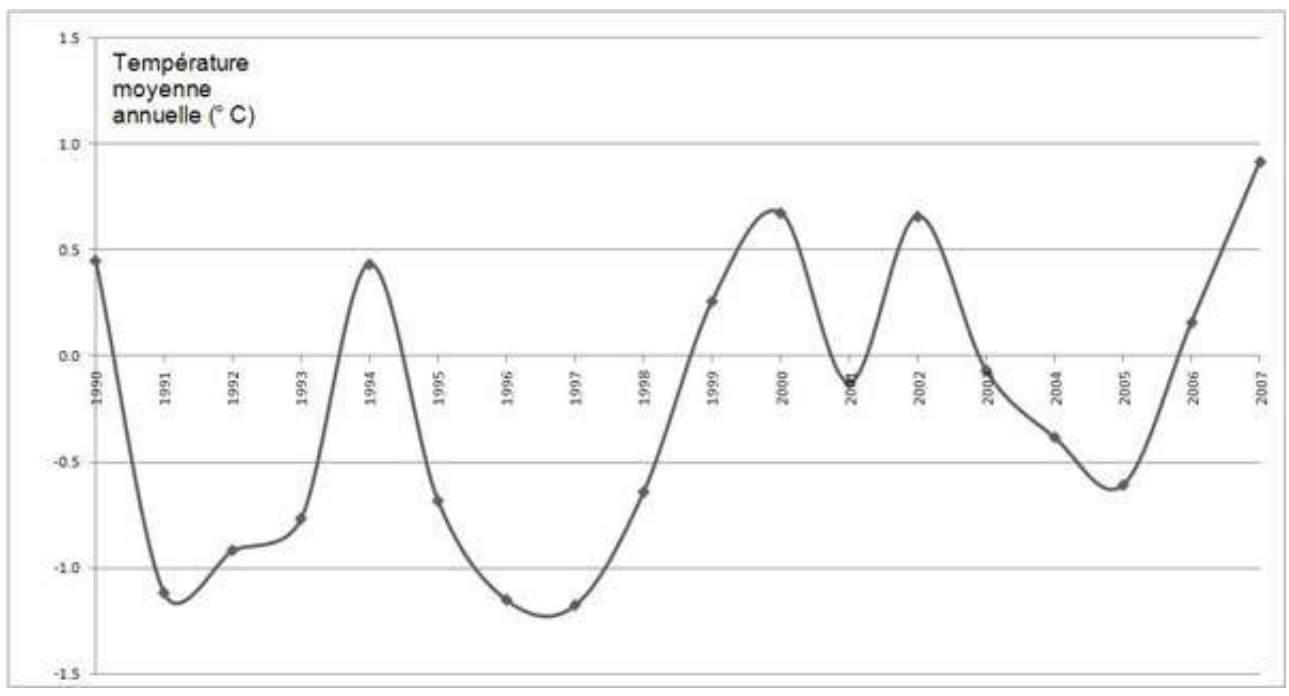

Figure 4 : Les précipitations enregistrées à la station météo Retitis pour l'intervalle 1990-2007.

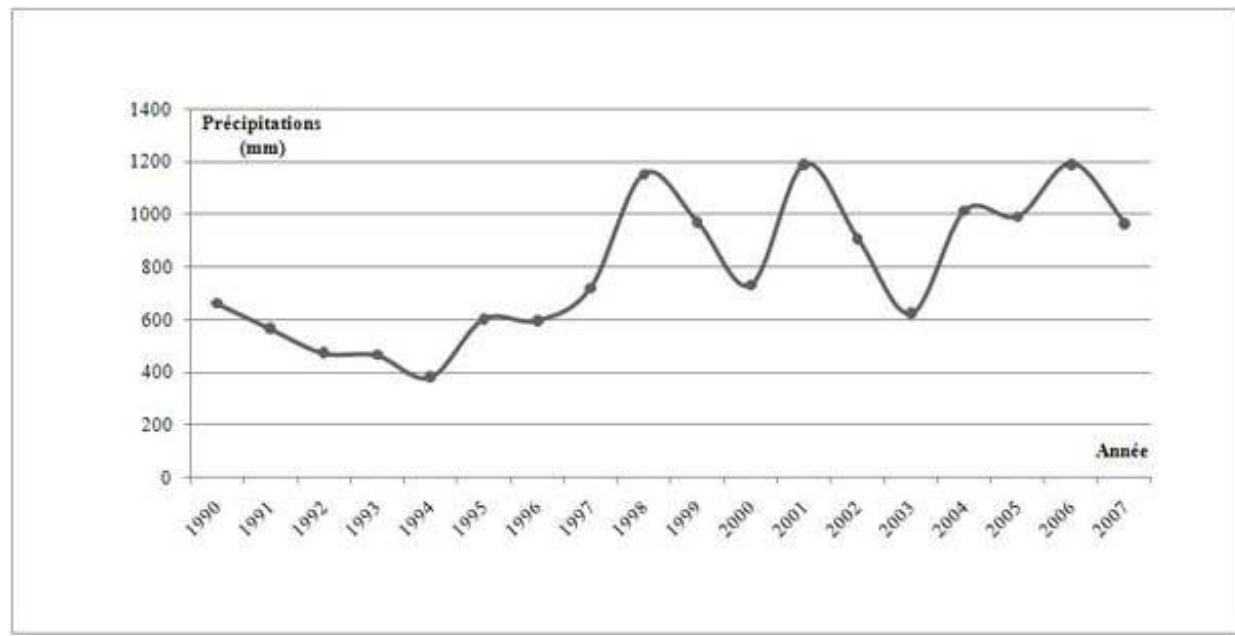

Dans la figure 5 nous avons sélectionné les jours qui ont enregistré des précipitations liquides dépassant $25 \mathrm{~mm}$. Pour la période 1990-2007, le graphique présente 52 jours où les précipitations liquides ont dépassé $25 \mathrm{~mm}$. Si on considère le seuil de $25 \mathrm{~mm} /$ jour comme nécessaire pour déclencher une coulée de débris, les 52 jours qui ont enregistré cette quantité de précipitation pourraient représenter autant de conditions favorables pour l'activité des coulées de débris. 
Figure 5 : Les jours avec plus de $25 \mathrm{~mm}$ de précipitations liquides enregistrés à la station météo Retitiş entre 1990 et 2007

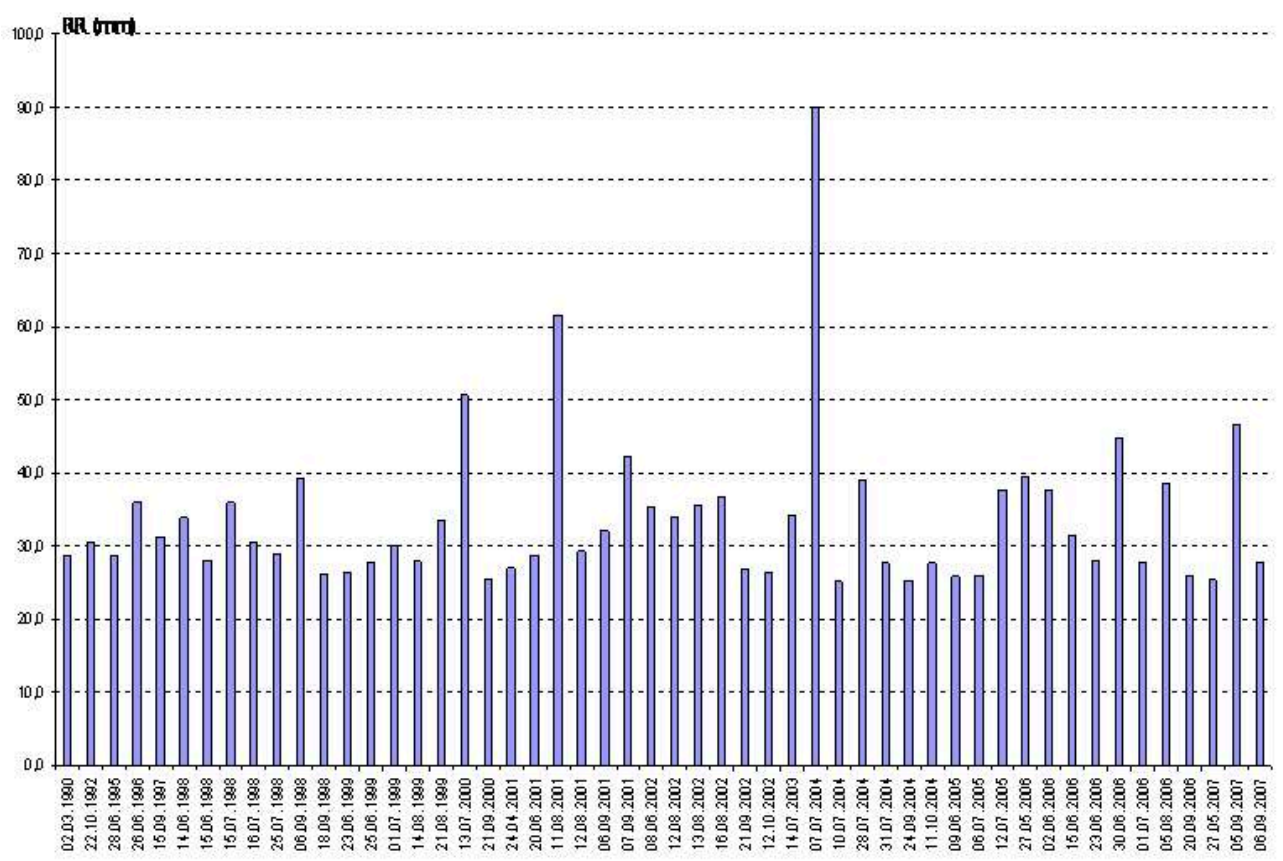

8 Dans les étages alpin (1900-2100 m) et subalpin (1700-1900), le rôle de protection des forêts contre l'érosion est diminué du fait de la raréfaction des arbres. La limite supérieure de la forêt d'épicéa (Picea abies) et de pin cembro (Pinus cembra) se situe vers 1650-1700 m. L'étage subalpin est constitué des arbustes tels que le pin de montagne (Pinus mugo), les myrtillers (Vaccinium myrtillus), les genévriers (Juniperus communis), etc.

9 Sur le cône du Negoiul Românesc, les surfaces affectées par l'activité minière sont situées vers la limite supérieure de la forêt et dans la partie inférieure de l'étage subalpin. A ces altitudes, la morphodynamique actuelle est dominée par l'activité des coulées de débris. Les quantités de précipitations liquides extrêmes (> $25 \mathrm{~mm}$ par jour), les versants pentus de plus de $20^{\circ}$ et les dépôts de terrils constitués de matériaux de granulométrie et composition pétrographique hétérogènes non-consolidées, constituent les facteurs qui favorisent ce type de processus. Des glissements de grande ampleur ont contribué aussi à la mobilisation d'une grande partie des terrils. A ces processus s'en rajoutent d'autres: le ruissellement, les désagrégations mécaniques causées par le gel/dégel, les processus fluviatiles dans les lits des rivières, etc.

\section{Un milieu forestier affecté par les activités minières}

Quelques précisions concernant l'historique des exploitations du soufre seront présentées ici brièvement, afin de mieux comprendre les zones du massif du Calimani transformées par l'activité minière.

11 Vers 1854, le géologue P. J. Kremnizki fut le premier qui décrivit le soufre natif d'origine volcanique découvert dans le massif du Calimani. A l'époque, sa découverte fut très appréciée par les fabricants locaux d'allumettes soucieux de s'approvisionner en matière première moins coûteuse que celle provenant de Sicile. 

1953 d'après les témoignages des habitants du village Gura Haitii (situé vers le N, à 15 $\mathrm{km}$ de la zone d'exploitation du soufre).

Plus tard dans les années soixante, les travaux de prospection (66 forages totalisant une longueur de $19218 \mathrm{~m}$ ) confirmaient la présence d'un important gisement de soufre sur les flancs du cône Negoiul Românesc. En réalité, le soufre était concentré plutôt à proximité de la surface, dans des fissures, ou imprégné dans des pyroclastites altérées et des laves andésitiques (photo 1).

Photo 1 : Soufre natif dans la carrière du Negoiul Romanesc; il se présente à l'état presque pur, (photo en haut) ou imprégné dans les pyroclastites altérées (photo en bas)

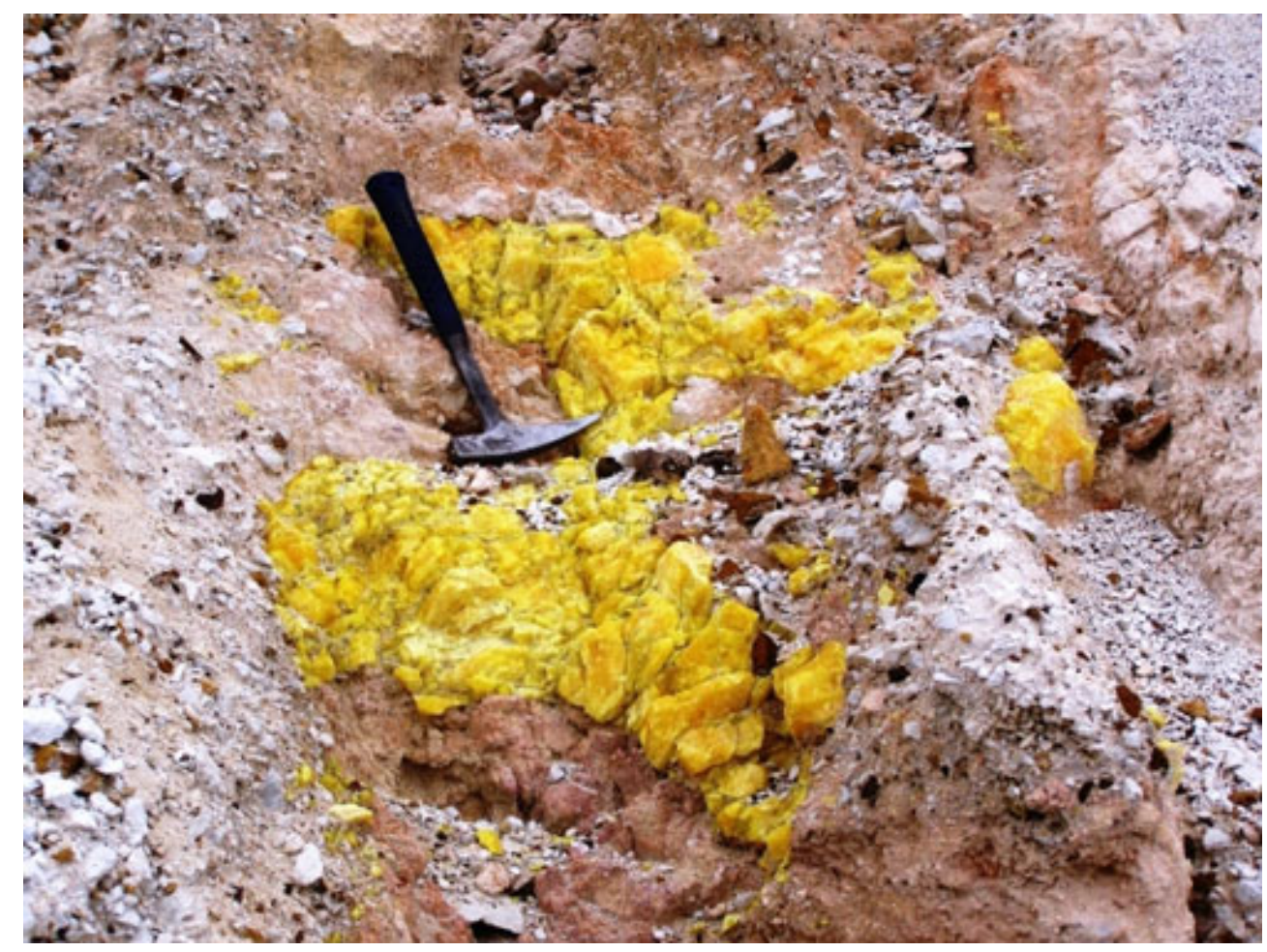




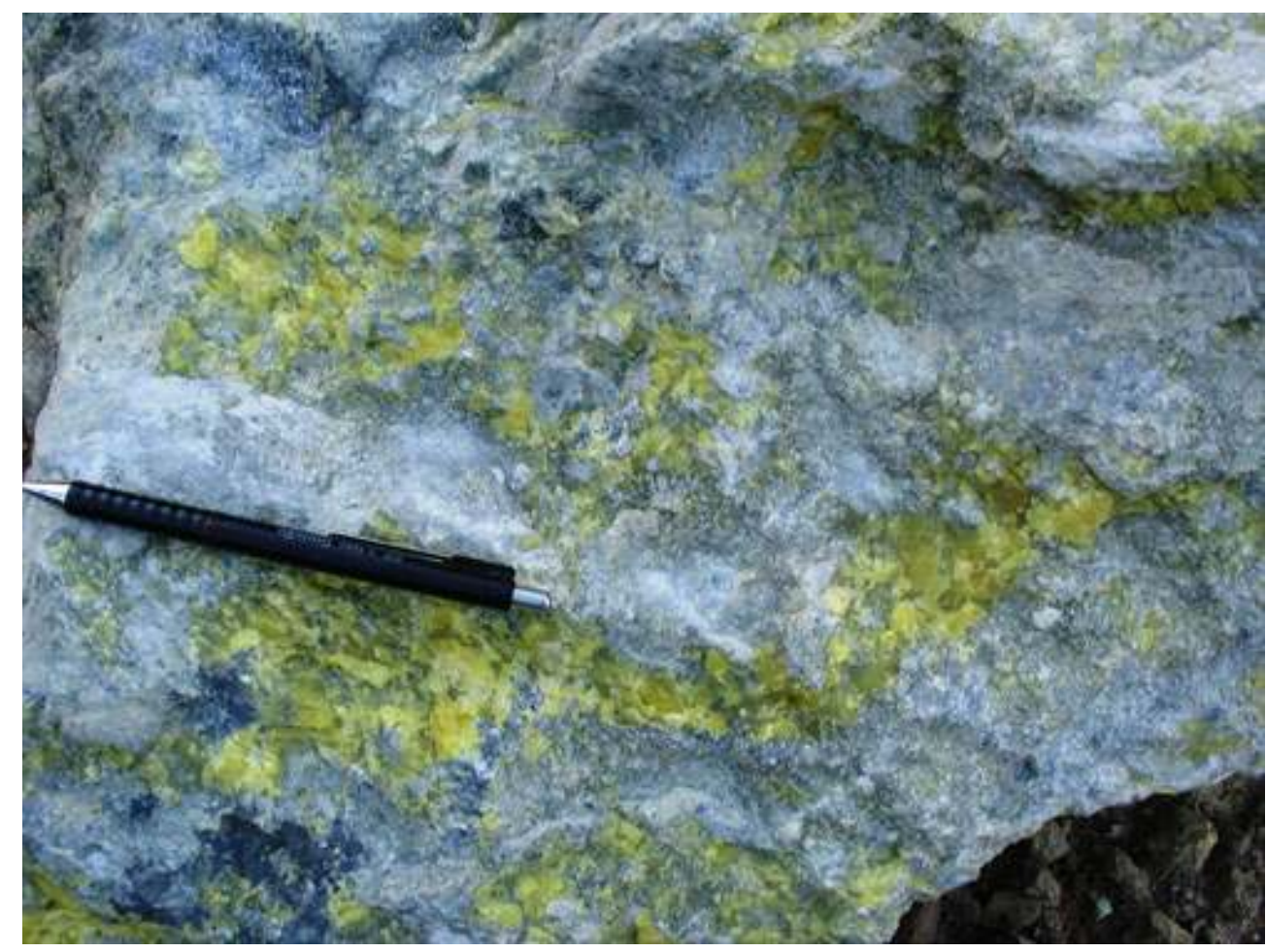

Initialement, on cherchait des modalités diverses de valoriser les oxydes de $\mathrm{Fe}$ accompagnant le soufre dans le gisement. Après plusieurs essais infructueux pour extraire les oxydes de $\mathrm{Fe}$, les recherches ont continué pour trouver la technologie appropriée afin de récupérer le soufre. Sous la pression des dirigeants communistes, les rapports des géologues "devaient” confirmer à tout prix la présence d'un gisement de soufre exploitable. Dans leur optique, un grand gisement de soufre, exploitable sur le territoire national, aurait pu réduire les importations de cette matière première de base pour l'industrie chimique subventionnée par le régime communiste.

15 A la suite des travaux de prospection effectués entre 1962 et 1972, les études géologiques ont délimité le périmètre du gisement de soufre exploitable. Les réserves ont été estimées à environ 30 millions tonnes de minerai contenant du soufre d'une concentration de $19,79 \%$ et 31,3 millions tonnes à $19,65 \%$ de soufre dans le minerai. Dans ces conditions, on prévoyait l'extraction d'environ 2,5 millions tonnes de minerai par an, quantité qui couvrait les besoin en soufre de l'industrie chimique nationale.

La décision de mise en exploitation du gisement a été prise en 1973. Très vite les travaux de construction des bâtiments (usine de préparation du soufre, habitations pour les ouvriers, bassins de décantation, etc.) et de l'infrastructure (route en béton, réseau d'électricité, etc.) ont modifié profondément la morphologie des versants et les écosystèmes de cette zone.

17 Les gisements de soufre exploités pendant la période 1971-1997 ont été vite épuisés, rendant cette activité peu rentable économiquement. Plusieurs essais visant à améliorer la technologie d'extraction du soufre ont été effectués mais sans aboutir à des résultats satisfaisants.

18 La chute du régime communiste en décembre 1989 a amené des changements profonds en ce qui concerne la poursuite des travaux d'exploitation dans la carrière du Negoiul Românesc. Un arrêt temporaire des travaux a été initialement imposé entre 1985 et 
1992. Après, en 1992 les travaux d'une ampleur plus réduite ont recommencé, continuant jusqu'en 1997, quand la carrière a été définitivement fermée.

Le nombre d'employés qui ont travaillé pour l'exploitation du soufre du Calimani a varié pendant la période d'activité. Si au début de l'exploitation on ne comptait que quelques centaines d'employés, leur maximum a été atteint durant les années '80 avec environ 1500 d'employés travaillant dans la carrière et dans l'usine de préparation du soufre. En 1998, par l'ordonnance gouvernementale 22/1997, tous ces employés se sont retrouvés sans travail. La plupart d'entre eux (plus de 80\%) habitaient les villages et villes avoisinantes (Gura Haitii, Neagra şarului, şaru Dornei, Vatra Dornei, etc.), les autres étaient originaires des départements Botoşani, Bistrita, etc. Tous ont dû soit devenir chômeurs, soit accepter une reconversion professionnelle et s'orienter vers d'autres domaines économiques d'intérêt local (exploitations forestières, agriculture, construction des bâtiments, tourisme rural). L'ouverture des frontières du pays a favorisé l'émigration vers l'Europe de l'Ouest (Espagne, Italie, France, Allemagne, Irlande, etc.), les Etats Unis ou le Canada d'une grande partie des anciens mineurs de la carrière de soufre du Calimani.

Les aménagements réalisés pour exploiter cette ressource n'ont jamais tenu compte de l'impact qu'ils pouvaient avoir sur l'environnement. Ainsi, de graves erreurs on été commises au moment du choix de l'emplacement des terrils. Sur les versants pentus, à proximité des carrières, les matériaux des terrils ont été déposés de manière chaotique, sans respecter les consignes des géologues de réaliser plusieurs plateformes en gradins (photo 2).

21 Le versant nord du Negoiul Românesc a été recouvert par des dépôts constitués de matériaux provenant de la carrière. La pression due à surcharge et à la présence de l'eau infiltré à travers les terrils jusqu'au contact entre les deux couches ont favorisé le déclenchement des glissements de terrain en 1975, 1978, 1979, 1982 (Bojoi et Brândus, 1984, 1985). 
Photo 2 : Terrils déposés à proximité de la carrière, sur le versant ouest du Negoiul Românesc ; les coulées de débris formées sur les pentes des accumulations ont transporté des matériaux en les déposant en aval dans la forêt ou dans le lit de la rivière Dumitrelul.

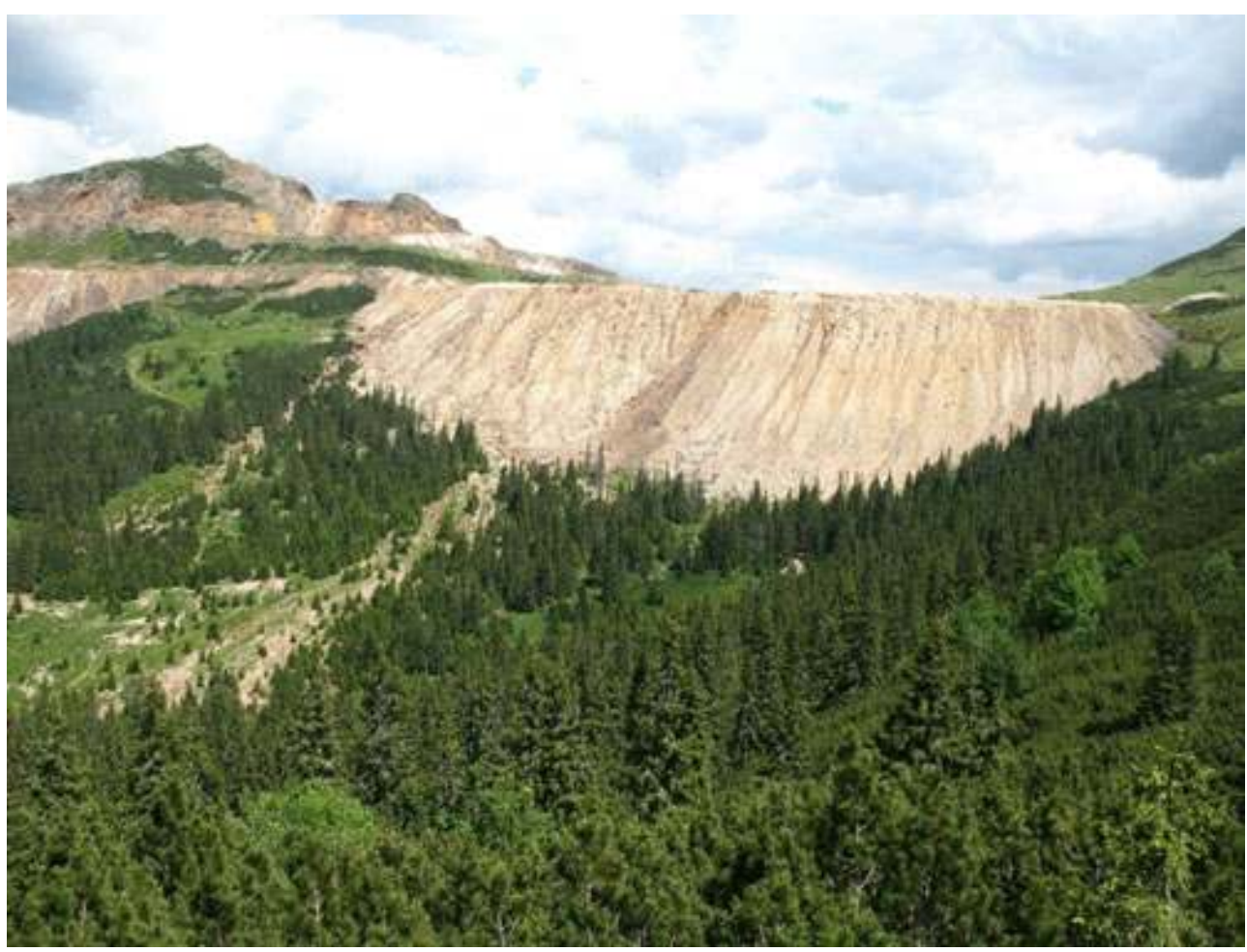

Les explosions utilisées dans la carrière ont contribué en grande mesure à la déstabilisation des terrils et au déclenchement de ces glissements. Ultérieurement, entre les corps des glissements, des coulées de débris ont transporté des quantités importantes de matériaux qui ont été déposés en aval sur les pentes plus faibles, dans les lits des rivières ou derrière les barrages construits pendant les années '80 (photo 3).

Sur les versants du Negoiul Românesc, les forêts de conifères (épicéa, pin cembro) et les arbustes de l'étage subalpin (pins de montagne, myrtillers, etc.) ont subi un fort impact des activités minières. Plus de 400 hectares de forêts ont été totalement coupés pour céder la place aux carrières, terrils, bâtiments, chemins d'accès, etc. En plus, la pollution atmosphérique, celle des sols et des eaux ont augmenté les superficies des forêts et des arbustes affectés. La mobilisation des terrils par de divers processus géomorphologiques (glissements, coulées de débris, écoulements hyperconcentrés, ruissellement, etc.) a affecté aussi de vastes zones boisées, situés en aval de la carrière et des terrils. 
Photo 3 : La surface des glissements produits en 1975, 1978, 1979, 1982 dans les terrils déposés sur le versant nord du Negoiul Românesc ; sur plusieurs $\mathrm{km}^{2}$, la forêt a été détruite par ces glissements et les coulées de débris formés ultérieurement ; depuis une vingtaine d'années, les corps des glissements, plus stables que les surfaces affectées par les coulées de débris, ont été colonisés avec des épicéas, bouleaux, peupliers ou saules marsault (photo en haut) ; les matériaux transportés par les coulées de débris ont été déposés sur les pentes réduites, dans les lits des rivières et derrière le barrage construit en aval dans la vallée du Pinul (photo en bas)
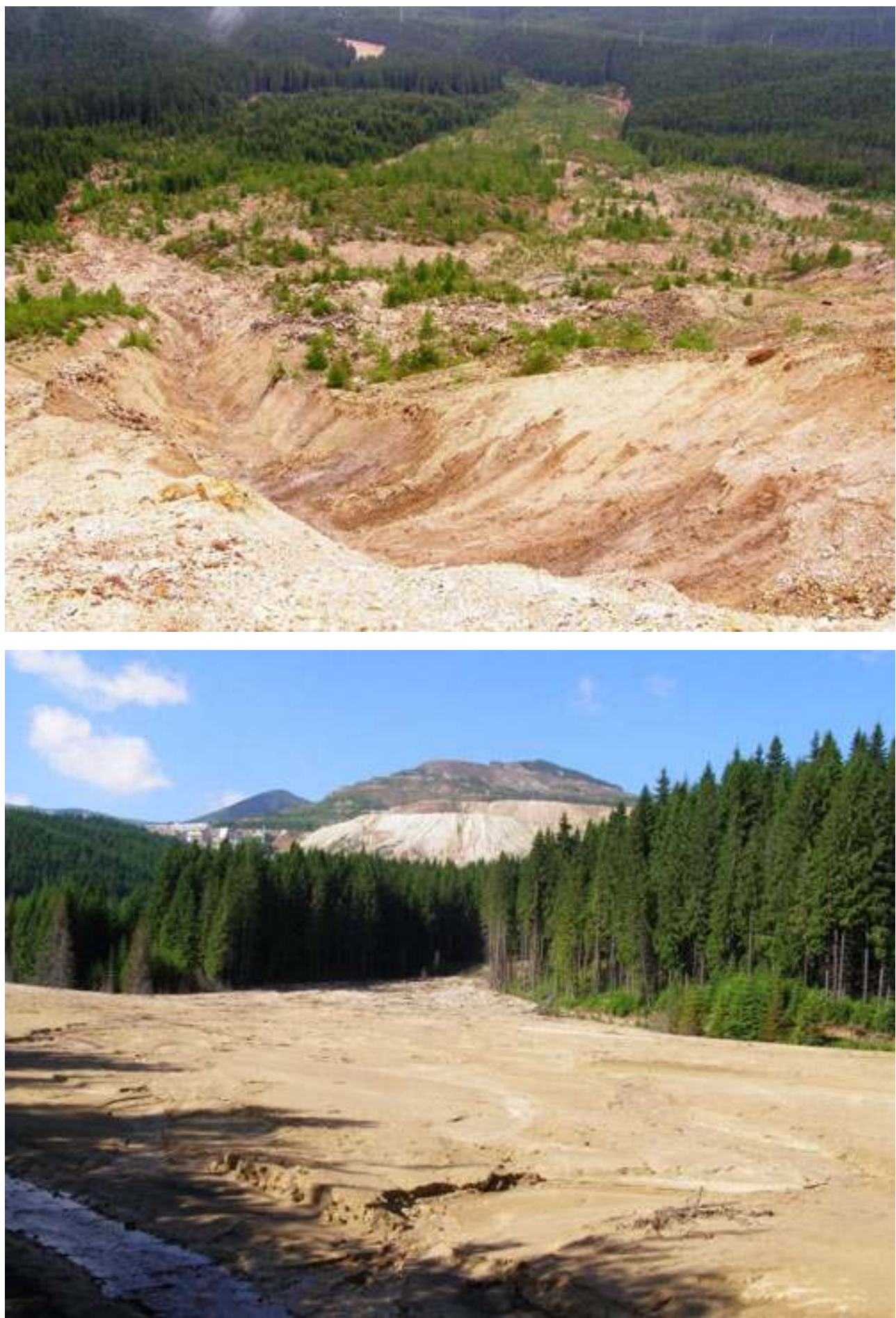


\section{L'instabilité morphodynamique actuelle des terrils et de la carrière}

Dans une première phase de notre étude, les cartes topographiques $1 / 25000$ et $1 / 5000$ de la zone d'étude ont été scannées et géoréférencées. En utilisant le logiciel ArcGIS 9, deux modèles numériques de terrain (MNT) d'une résolution de 5 et $10 \mathrm{~m}$ ont été réalisés à partir des courbes de niveau numérisées sur les cartes.

Utilisant les orthophotoplans de la mission 2005 de l'Agence Nationale du Cadastre et de la Publicité Immobilière, nous avons analysé l'occupation du sol actuelle en délimitant des polygones selon une légende préétablie.

La carte de l'occupation du sol a été validée sur le terrain (juillet 2007, juin et août 2008), certaines modifications étant apportées là où la photo-interprétation ne correspondait pas avec la réalité. Sur le MNT obtenu à partir des courbes de niveau numérisées, nous avons ensuite superposé la carte de l'occupation du sol (figure 6) réalisée à partir des orthophotoplans des observations de terrain.

Les cartes topographiques noir et blanc à l'échelle $1 / 25000$, nous ont fourni des informations concernant l'occupation du sol pendant les années soixante. A cette époque, les arbustes occupaient totalement le sommet du Negoiul Românesc, descendant sur les versants jusqu'à l'altitude d'environ $1700 \mathrm{~m}$ où s'étendait la forêt.

La carte de l'occupation du sol actuelle de la figure 6 présente une situation bien différente de celle des années soixante. Sur cette carte, les zones de la carrière (65 hectares), des terrils (120 hectares) et bâtiments (plateforme industrielle pour la préparation du soufre, habitations pour les ouvriers totalisant 50 hectares) sont entourées de forêts plus ou moins affectées. Les forêts détruites par l'activité des processus géomorphologiques tels que les glissements de terrain et les coulées de débris sont situées en aval des terrils. Les forêts conservées, les arbustes et les prairies situés loin de la carrière et des terrils n'ont pas subi directement la pression des activités minières, ou celle des processus géomorphologiques. Cependant, sur une vaste surface autour de la zone minière, la pollution atmosphérique ( $\mathrm{H} 2 \mathrm{~S}$, SO2, les poussières) et les pluies acides formées (Dị̧oiu, 2002, Popa et Kern, 2008) ont affecté la végétation forestière et arbustive. 
Figure 6 : Orthophotoplans de 2004 avec la zone d'étude (en haut) et carte de l'occupation du sol de la zone affectée par l'activité minière (en bas) : 1 - sommets ; 2 - courbe de niveau ; $3 a$ - cours d'eau temporaire, $3 \mathrm{~b}$ - cours d'eau permanent ; 4 - carrière ; 5 - terrils ; 6 - usine et autres bâtiments ; 7 - forêt partiellement affectée par l'activité minière ; 8 - forêt détruite par les glissements de terrain et les coulées de débris sur les terrils ; 9 - forêt conservée ; 10 reboisements ; 11 - végétation arbustive ; 12 - prairies subalpines.
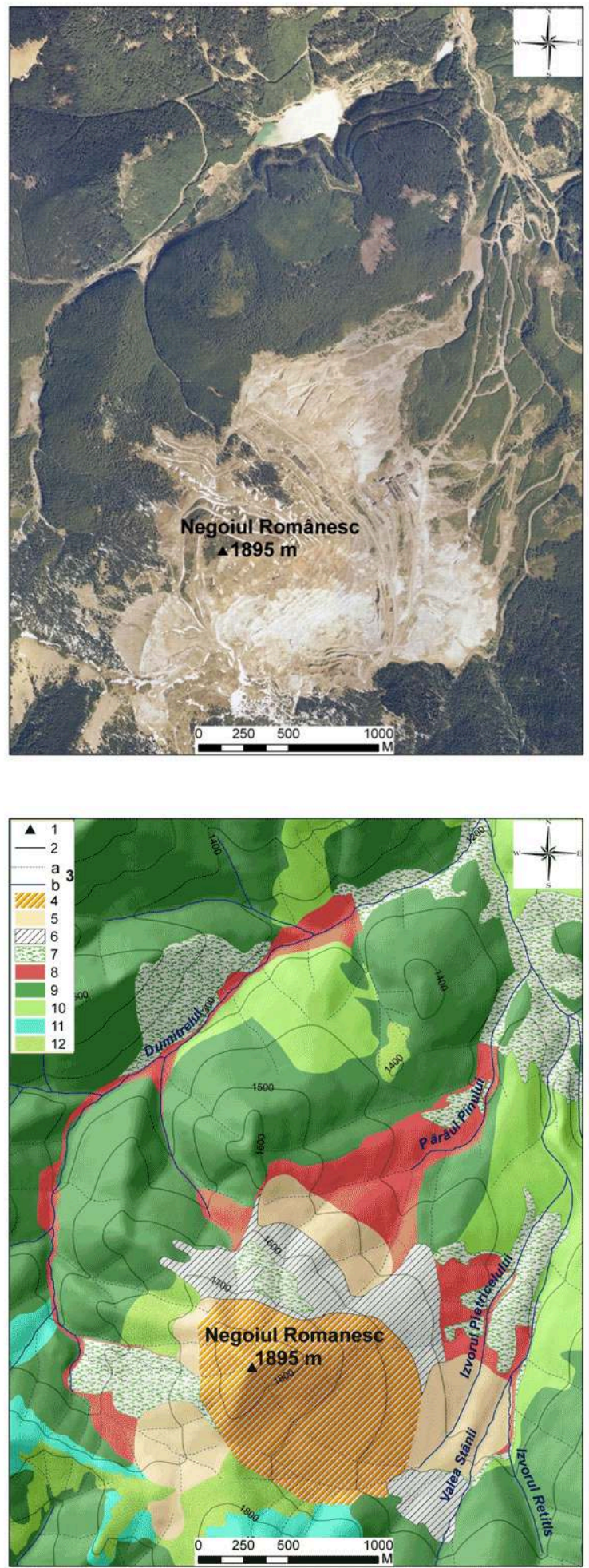
Tout au long des cours d'eau drainant la zone minière, une pollution intense des eaux et des sols a déterminé la mort d'un grand nombre d'arbres, en affectant partiellement d'autre situés à proximité du lit de la rivière. En plusieurs points, le $\mathrm{pH}$ actuel des eaux de ces rivières a été déterminé, les analyses indiquant des valeurs inférieures à 3 , ce qui explique l'absence totale de la faune dans les rivières et la mort des arbres situés à proximité.

Dans plusieurs sites, des analyses dendrogéomorphologiques ont été effectuées sur les arbres localisés à proximité de ces lits des ruisseaux pollués Pinul et Dumitrelul. Elles nous ont confirmé le fait qu'à partir des années soixante-dix, les cernes annuels sont de plus en plus réduits, ce qui correspond avec la période quand la pollution a commencé d'affecter les arbres. Les sédiments qui ont endommagé les troncs et les racines des arbres, ont laissé des cicatrices qu'on a pu reconnaître à l'extérieur ou à l'intérieur du bois en analysant des sections transversales ou des carottes.

31 Au début des années quatre-vingt, sur les ruisseaux Pinul et Dumitrelul deux barrages ont été construits en aval des terrils, dans le but de stabiliser les sédiments transportés par les coulées de débris ou les coulées hyperconcentées. Ils sont actuellement colmatés et risquent de déborder si d'autres événements de grande ampleur se reproduiront. En 2007, le niveau actuel des sédiments accumulés derrière ces barrages a été déterminé en utilisant un théodolite. La topographie initiale (indiquée par les cartes topographiques 1/5000) et le niveau actuel (déterminé à l'aide des mesures topographiques) des sédiments dans le barrage nous ont permis de calculer les volumes des matériaux accumulés dans les deux barrages. Ainsi, dans le barrage Dumitrelul, le volume de sédiments accumulé jusqu'en 2007 dépassait $25500 \mathrm{~m} 3$, le taux d'accumulation des matériaux calculé étant estimé à environ $1430 \mathrm{~m} 3$ par an. Dans le cas du barrage Pinul, le volume accumulé jusqu'en 2007 était de 91100 m3, avec le taux d'accumulation estimé à $3250 \mathrm{~m} 3$ par an.

De nos jours, plus d'une décennie après l'arrêt définitif des travaux, les zones minières abandonnées n'ont bénéficié d'aucune réhabilitation. La carrière et les terrils ont été et continuent d'être très instables morphologiquement, et constituent une menace permanente pour les écosystèmes forestier et aquatique, ainsi que pour les éléments d'infrastructure (route d'accès vers la partie centrale du massif, barrages de décantation et de rétention des résidus stériles ou des sédiments provenant des terrils).

Durant l'été 2008 les travaux de réhabilitation des secteurs affectés par l'activité minière ont démarré, 11 ans après l'arrêt définitif de l'activité minière. L'Etat Roumain finance les travaux avec des fonds provenant des emprunts à la Banque Mondiale. Mais, ces interventions pour réhabiliter et écologiser la zone minière arrivent trop tard, les conséquences de la pollution et de l'instabilité morphodynamique sur l'infrastructure, la forêt et l'eau des rivières étant dramatiques. Les surfaces affectées par cette exploitation minière irraisonnable et qui nécessitent la réhabilitation sont trop vastes, ces fonds ne seront pas suffisants pour couvrir intégralement les travaux. Analysant l'ampleur de l'impact environnemental, on peut affirmer sans hésitation qu'une stabilité totale et une pollution nulle ne se réaliseront jamais par des travaux de ce type. Les solutions de réhabilitation proposées ne respectent pas en totalité les consignes concernant les activités acceptées dans le Parc National, organisation créée en 2000 qui gère actuellement une grande partie de la zone d'exploitation minière. Dans ce cas, les interventions autorisées ne devront pas contribuer à créer d'autres dérèglements environnementaux. 
l'équitation contribuera à la diversification de l'offre et devra attirer plus de touristes. Le géotourisme pourra valoriser les potentiels des géosites et géomorphosites d'origine naturelle ou anthropique présents en grand nombre dans le massif du Calimani. La carrière et des terrils devront être stabilisés et aménagés pour les touristes selon les modèles français, suisses allemands ou italiens des zones affectées par les exploitations minières. L'intérêt de la carrière est lié au fait qu'elle permet la découverte de la structure interne du cône volcanique Negoiul Românesc, des roches le constituant, des conduits et des grottes pseudo-volcano-karstiques qui traversent ces structures, les conditions de gisement du soufre, etc. Les terrils représentent un laboratoire géomorphologique à ciel ouvert où l'on peut observer et suivre à l'échelle réelle la variété des processus géomorphologiques qui y agissent et les changements morphologiques annuels. Les classes vertes pour les élèves et les étudiants ainsi que les sorties guidées organisées pour le grand public pourront bénéficier de la présence de ces géosites originels. Elles éveilleront davantage l'intérêt pour la science en général, pour la volcanologie, la pétrographie et la géomorphologie volcanique en particulier. Cette forme de vulgarisation scientifique contribuera ensuite au développement touristique et économique de cette région dont les potentiels naturel et anthropique sont actuellement peu valorisés.

\section{Conclusions}

La présente étude a permis d'évaluer l'impact des activités minières sur la morphologie de la partie centrale du massif du Calimani. La carte de l'occupation du sol montre l'état actuel et l'extension des changements spatiaux intervenus suite à l'exploitation du soufre en carrière, commencée durant les années '70 au XXème siècle sur les versants du cône Negoiul Românesc.

Les responsables communistes qui ont suivi les travaux d'exploitation en carrière du soufre, n'ont jamais tenu compte de l'impact que cette activité aurait pu avoir sur les éléments de l'environnement (la morphologie des versants et des lits de rivière, les eaux, les sols, les forêts, la faune, etc.). De nos jours, la carrière et les terrils se caractérisent par une instabilité morphologique élevée, étant en même temps une source permanente de pollution atmosphérique, de l'eau ou des sols.

Actuellement, l'inconscience des anciens dirigeants communistes coûte cher à l'Etat, qui doit financer les travaux de réhabilitation, en faisant appel à des emprunts à la

Revue Géographique de l'Est, vol. 49 / 1 | 2009 
Banque Mondiale. Mais, vu l'ampleur actuelle de la morphodynamique et la pollution, à notre avis même les travaux de réhabilitation ne pourront effacer totalement les traces de l'impact minier dans la région. Dans l'avenir, on assistera donc à une «réhabilitation naturelle» pour une grande partie des terrils et pour la carrière en totalité. Les futurs aménagements dans cette zone pourront favoriser l'activité touristique (randonnée, géotourisme etc.) et contribuer indirectement à son développement économique.

\section{BIBLIOGRAPHIE}

Bojoi I., Brandus C. (1984). - « Influente antropice asupra modelarii reliefului Masivului Caliman ». Studii si Cercetari de Geologie, Geofizica si Geografie, seria Geografie, tomul XXXI, p. 14-18.

Bojoi I., Brandus C. (1985). - « Considérations sur la morphodynamique actuelle du Massif des Călimani (Carpates Orientales) ». Analele ştiinţifice ale UniversităŢii „Al. I. Cuza”, Iași, tomul XXXI, seria II b, Geologie-Geografie, p. 67-73.

Ditoiu V. (2002). - « Contributii privind stabilirea impactului produs de activitatile miniere asupra ecosistemelor din zona Calimani ». Résumé de la thèse de doctorat, Universitatea Tehnica "Gheorghe Asachi", Facultatea de Chimie Industriala, Iasi, 30 p.

Karátson D. (1996). - « Rates and factors of stratovolcano degradation in a continental climate: a complex morphometric analysis for nineteen Neogene / Quaternary crater remnants in the Carpathians ». Journal of volcanology and geothermal research, $n^{\circ} 73$, p. 65-78.

Pécskay Z., Edelstein O., Seghedi I., Szakács A., Kovács M., Crihan M., Bernad A. (1995). -

« K-Ar dating of Neogene-Quaternary calc-alkaline volcanic rocks in Romania ». Acta Vulcanologica, vol. 7, n², p. 53-61.

Pécskay Z., Lexa J., Szakács A., Balogh K., Seghedi I., Konečný V., Kovács M., Márton E., Kaličiak M., Széky-Fux V., Póka T., Gyarmati P., Edelstein O., Roşu E., Žec B. (1995). - «Space and time distribution of Neogene-Quaternary volcanism in the Carpatho-Pannonian Region ». Acta Vulcanologica, vol. 7, n², p. 15-28.

Popa I., Kern Z. (2008). - « Long-term summer temperature reconstruction inferred from treering records from the Eastern Carpathians ». Climate Dynamics, (en cours de publication).

Seghedi I.,Szakács A., Pécskay Z., Mason P. R. D. (2005). -« Eruptive history and age of magmatic processes in the Calimani volcanic structure (Romania) ». Geologica Carpatica, vol. 56, n¹, p. 67-75.

Szakács A., Seghedi I. (2000). - « Large volume volcanic debris avalanche in the East Carpathians, Romania ».In : Leyrit H., Montenat C., Volcaniclastic Rocks from magmas to sediments, Gordon and Breach Science Publishers, Amsterdam, p. 131-150.

Szakács A., Krézsek C. (2006). -« Volcano-basement interaction in the Eastern Carpathians: explaining unusual tectonic features in the Eastern Transylvanian Basin, Romania». Journal of Volcanology and Geothermal Research, vol. 158, p. 6-20. 


\section{RÉSUMÉS}

En Roumanie, la seuleexploitation du soufre en carrière a été ouverte en 1973 dans le secteur du Negoiul Romanesc situé dans la partie centrale du massif du Calimani (Carpates Orientales roumaines). Mais, à cause de la faible rentabilité, cette activité a été arrêtée définitivement en 1997. Actuellement, les versants du cône transformés en carrière et terrils sont très instables, caractérisés par une morphodynamique active (coulées de débris, glissements de terrain, etc.). Utilisant des orthophotoplans, des cartes topographiques $1 / 25000$ et $1 / 5000$ et les informations provenant des observations de terrain, des sources bibliographiques diverses ou des témoignages, nous avons réalisé une carte actuelle de l'occupation du sol de cette région. Sur cette carte, on observe l'extension spatiale des zones de carrière, des terrils ou de la plateforme industrielle réalisée au détriment des forêts. En aval des terrils, les glissements de terrain et les coulées de débris ont affecté les forêts. Des mesures topographiques ont été effectuées afin de déterminer la vitesse de remplissage des sédiments transportés par les coulées de débris et déposés derrière les barrages construits pour stopper leur progression. Les valeurs obtenues indiquent des vitesses de remplissage de $1430 \mathrm{~m}^{3}$ par an dans le cas du barrage Dumitrelul, respectivement $3250 \mathrm{~m}^{3}$ par an pour le barrage Pinul. Des travaux de réhabilitation des terrils, de la carrière et de la plateforme industrielle ont commencé en juillet 2008, 11 ans après l'arrêt définitif de l'activité minière.

In Romania, the onlyopencast sulphur mining was begun in 1973 in the Negoiul Românesc area, situated in the central part of the Călimani massif (Romanian Oriental Carpathians). In 1997 the mining activity ceased for good because of the low profitability. At the moment, the volcanic cone slopes that were turned into quarry and spoil heaps are extremely unstable, and they are characterized by active morphodynamics (debris flows, landslides, etc.). Based on the use of orthophotoplans, topographical maps $(1 / 25000$ and 1/5000) and field observations, various bibliographical sources or accounts, we have made a present-day land use map of this area. This map shows the spatial expansion of the quarry, the spoil heaps and the industrial platform that replaced the forest. Downstream the spoil heaps the forests were affected by landslides and debris flows. Topographical measurements have been carried out in order to determine the accumulation rate of the sediments transported by the debris flows and laid beyond the dams that were built in order to stop them. The results show an accumulation rate of $1430 \mathrm{~m}^{3}$ per year for the Dumitrelul dam, respectively $3250 \mathrm{~m}^{3}$ per year for the Pinul dam. In July 2008, 11 years after the definitive cease of the mining activity, rehabilitation work was begun in the spoil heaps, the quarry and the industrial platform.

Der einzige Schwefel-Tageabbau in Rumänien wurde im Jahre 1973 im Gebiet des Negoiul Românesc, im zentralen Teil des Călimani-Gebirges (Rumänische Ostkarpaten), eröffnet. Wegen der geringen Rentabilität wurde jedoch der Abbau 1997 endgültig eingestellt. Z.Z. sind der in einen Steinbruch umgewandelte Kegel und die Halden sehr unbeständig, so dass sie von aktiven morphodynamischen Prozessen erfasst worden sind (Schuttfließen, Erdrutschungen u.a.). Durch Verwendung von Orthophotoplänen, topographischen Karten im Maßstab 1:25.000 und 1: 5.000, durch Geländestudien und Auswertung verschiedener Informationsquellen und Berichte wurde die aktuelle Nutzungskarte des Gebietes erstellt. Man erkennt die Ausdehnung der Steinbrüche, der Abraumhalden und der Industrieplattformen, die an Stelle des Waldes angelegt wurden. Im unteren Bereich haben Erdrutsche und Schuttströme den Wald verdängt. Es wurden topographische Messungen gemacht, um die Geschwindigkeit festzustellen, mit der sich die hinter Staumauern angelegten Becken mit Gesteinsschutt auffüllen, die diesen zurückhalten sollen. Die ermittelten Werte zeigen eine Auffüllgeschwindigkeit von $1430 \mathrm{~m}^{3}$ pro Jahr am Dumitrelul-Stauwehr und $3250 \mathrm{~m}^{3}$ am Pinul Stauwehr. Die Rehabilitierungsarbeiten an den 
Halden, Steinbrüchen und Industrieplattformen haben im Juli 2008 begonnen, elf Jahre nach der vollständigen Einstellung des Abbaus.

\section{INDEX}

Keywords : Călimani massif (Romania), debris flows, landslides, morphodynamic instability, quarry, spoil heaps

Schlüsselwörter : Călimani-Massiv (Rumänien), Erdrutsch, Halde, morphodynamische Instabilität, Schuttstrom, Steinbruch

Mots-clés : carrière, coulées de débris, glissements de terrain, instabilité morphodynamique, massif du Calimani (Roumanie), terrils

\section{AUTEURS}

\section{OLIMPIU TRAIAN POP}

Université Babes-Bolyai, Cluj-Napoca, Roumanie ; Université Blaise-Pascal, laboratoire CERAMAC, Clermont-Ferrand, France - olimpiu_p@yahoo.com

\section{NICOLAIE HODOR}

Université Babes-Bolyai, Cluj-Napoca, Roumanie - nicuhodor@yahoo.com

\section{VIRGIL SURDEANU}

Université Babes-Bolyai, Cluj-Napoca, Roumanie - surdeanu@geografie.ubbcluj.ro

\section{IOAN-AUREL IRIMUS}

Université Babes-Bolyai, Cluj-Napoca, Roumanie - irimus@geografie.ubbcluj.ro 